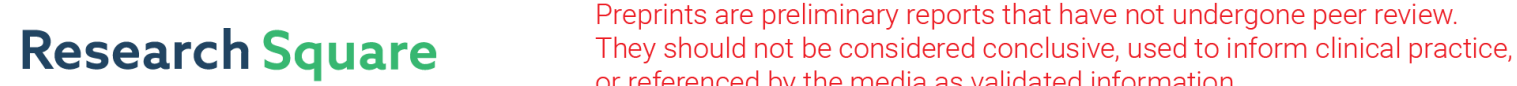 or referenced by the media as validated information. \\ Gait Recognition Analysis for Human Identification Analysis- A Hybrid Deep Learning Process
}

Mathivanan B ( $\nabla$ mathivananbcse@gmail.com )

Sri Ramakrishna Engineering College

Perumal P

Sri Ramakrishna Engineering College

\section{Research Article}

Keywords: emotions, walking styles, gait recognition, comparison analysis, statistical measurements and classification

Posted Date: June 28th, 2021

DOI: https://doi.org/10.21203/rs.3.rs-549846/v1

License: (1) This work is licensed under a Creative Commons Attribution 4.0 International License. Read Full License 


\title{
Gait Recognition analysis for Human Identification Analysis- A Hybrid Deep Learning Process
}

\author{
$1 *$ Dr. B. Mathivanan, ${ }^{2}$ Dr. P. Perumal \\ $1 *$ Associate Professor, Department of Computer Science and Engineering, \\ Sri Ramakrishna Engineering College, \\ Vattamalaipalayam, N.G.G.O Colony Post, Coimbatore - 641022. \\ mathivananbcse@gmail.com \\ ${ }^{2}$ Professor, Department of Computer Science and Engineering, \\ Sri Ramakrishna Engineering College, \\ Vattamalaipalayam, N.G.G.O Colony Post, Coimbatore - 641022. \\ perumalpcse@gmail.com
}

\begin{abstract}
Gait is an individual biometric behavior which can be detected based on distance which has different submissions in social security, forensic detection and crime prevention. Hence, in this paper, Advanced Deep Belief Neural Network with Black Widow Optimization (ADBNN-BWO) Algorithm is developed to identify the human emotions by human walking style images. This proposed methodology is working based on four stages like preprocessing, feature extraction, feature selection and classification. For the pre-processing, contrast enhancement median filter is used and Hu Moments, GLCM, Fast Scale-invariant feature transform (F-SIFT), in addition skeleton features are used for the feature extraction. To extract the features efficiently, the feature extraction algorithm can be often very essential calculation. After that, feature selection is performed. Then the classification process is done by utilizing the proposed ADBNN-BWO Algorithm. Based on the proposed method, the human gait recognition is achieved which utilized to identify the emotions from the walking style. The proposed method is validated by using the open source gait databases. The proposed method is implemented in MATLAB platform and their corresponding performances/outputs are evaluated. Moreover, the statistical measures of proposed method are also determined and compared with the existing method as Artificial Neural Network (ANN), Mayfly algorithm with Particle Swarm
\end{abstract}


Optimization (MA-PSO), Recurrent Neural Network -PSO (RNN-PSO) and Adaptive Neuro Fuzzy Inference System (ANFIS) respectively.

Keywords: emotions, walking styles, gait recognition, comparison analysis, statistical measurements and classification.

\section{Introduction}

The number of devices connected to the Internet (IoT) is growing every year, resulting in the data they generate. Among these devices, smartphones play an important role due to the continuous increase in their functionality and user acceptance [1]. Therefore, security in these devices should be one of the key points to be considered in their development. Some of the latest techniques used to prevent unauthorized access to mobile devices are based on biometrics [2,3]. It involves the analysis and measurement of a person's different physical characteristics or behaviors with the aim of achieving recognition or identity. Biometric identification systems have evolved over time and with the development of technology. The most popular are fingerprint, facial recognition, retinal scan, palm geometry and voice recognition. However, other biometric types are being developed that are less invasive [4]. An example of this is recognizing people based on the characteristics of their style. There are several pros and cons to this sign along the way a person walk [5].

Its main advantages are that it allows an automatic, specific and intrusive identification (following the principles of "quiet computer" and is not disturbed by technology by the user) [6] because he or she is the only one carrying the device (which is usually a smartphone) or computer vision Will be registered for identification. Disadvantages include biometric methods such as fingerprints that are less accurate than others [7]. This can be solved by the continuous and periodic identification mentioned above. Data Models Used for User Detection User walking [8] (for example, $3 \mathrm{~s}$ as used in this paper), can always be tried and the performance of user-based identification methods can be enhanced if data models used for diagnostics are created. It takes a very short time to make the correct identification. This manual testing process is time consuming and fails to be properly identified by an expert. Artificial intelligence (AI) $[9,10]$ is used in the system to reduce diagnostic time and reduce human error.

To achieve the reliable and efficient diagnosis results, the automatic detection and diagnosis systems are developed. The machine learning, deep learning and optimizations are utilized to enable the automatic detection of human identification. Many different types of machine learning are available to detection of diseases such as Artificial 
Neural Network (ANN) [11], Bayesian Network and Support Vector Machine (SVM) [12] respectively. The machine learning algorithm may fail to provide efficient results for huge amount databases. Hence, the deep learning approach have been interesting to detect the human identification from the features like as Convolutional Neural Network (CNN) [13], Deep Neural Network (DNN) and Deep Belief Neural Network (DBNN). To achieve best prediction rate, the feature selection is an essential task in the detection phase. The feature selection is utilized with the consumption of metaheuristic algorithms such as Particle Swarm Optimization (PSO) [14], Firefly Algorithm (FA), Grey Wolf Optimization (GWO), Whale optimization Algorithm (WOA) [15] and so on. These algorithms may be fails in the convergence rate. Hence, the efficient detection method and feature selection method is developed in this paper.

\section{The main contribution and organization of the research}

* In this paper, ADBNN-BWO Algorithm is developed to identify the human emotions by human walking style images. This proposed methodology is working based on four stages such as pre-processing, feature extraction, feature selection and classification.

* For the pre-processing, contrast enhancement median filter is used and Hu Moments, GLCM, F-SIFT, and skeleton features are used for the feature extraction. To extract the features efficiently, the feature extraction algorithm can be often very essential calculation. After that, feature selection is performed.

* Then the classification process is done by utilizing the proposed ADBNN-BWO Algorithm. Based on the proposed method, the human gait recognition is achieved which utilized to identify the emotions from the walking style. The proposed method is validated by using the open source gait databases.

* The proposed method is implemented in MATLAB platform and their corresponding performances/outputs are evaluated. Moreover, the statistical measures of proposed method are also determined and compared with the existing method as Artificial Neural Network (ANN), Mayfly algorithm with Particle Swarm Optimization (MA-PSO), Recurrent Neural Network -PSO (RNN-PSO) and Adaptive Neuro Fuzzy Inference System (ANFIS) respectively.

The remaining part of the paper is organized as. Section 2 provides the detail review of existing works. The detail description of the proposed methodology is explained in section 3. The performance evaluations of the proposed methodology are given in section 4. The conclusion part of the paper is presented in section 6 . 


\section{Literature Review}

Many different methods are available to achieve the human identification process. Some of the methods are reviewed in this section.

Sanjeevkumar Angadi et al., [16] have introduced a combination of the Bayesian system that consists of two phases: the main phase is the human distinction based on the item, and the next phase is the human identification based on spatial features. First, the records were observed in the primary position, where the article was invented using the Viola Jones calculation. When the items are differentiated, the component extraction process is completed by using different balanced skeletons to extract specific features. Item Skeleton provides a potential and intuitive discussion that provides material approval and article integration. The Bayesian system adjusts to item-based features to recognize people. In the spatial-based human ID phase, spatial features were segmented and sent to a pedagogical system based on people recognition. For a long time, yields were obtained using fuzzy entropy to recognize people. The test composition of the proposed mixture was performed using the UCF-Crime database, and the default value of the measurements of the exhibition, especially the F1-score, accuracy and rating of $0.8820,0.8770$ and 0.9203 , separately.

Habiba Arshad et al., [17] introduced the Quartile Deviation of Normal Distribution (QDoND) for Human Extraction and the Bayesian model for the combination of highlights with the distribution of highlights and the best highlights resolution. First, during the pre-production phase, the best channel was selected and its operating stream was rated. Motion locations are separated by QDoND, which is then used for shape and surface component extraction. After a while, the extracted features were merged by the Bayesian model according to their comparative record. Finally, the best features based on PDs were selected and approved based on the best features using the multiglass support vector machine. Four explicit and appreciated datasets were used to evaluate the proposed structure, for example, according to AVA Multi-view gait (AVAMVG), Cassia A, Cassia B and Cassia C 100\%, 98.8\%, $87.7 \%$ And $91.6 \%$ separately. The results reveal that the proposed technique beats in contrast to existing strategies.

Siogian Choi et al., [18] have introduced a strong margin level integration with the technique for straight approval, which controls the impact of turbulent examples as much as envelope example discrimination power. To this end, measure the quality of the skeleton up to the physical balance of each edge. When viewing quality, create a standardized cost base between input exteriors and listed cases to prevent synchronization with cluttered examples. Here, two-level straight integration is used for the cost phase to find an envelope level discrimination score including 
comparison and margin. Ultimately, the nature of an experiment is controlled by a weighted dominant area. It enhances the dosage against false skeletal evaluation results by assigning different loads to each envelope depending on the score. This method beats sophisticated techniques in three general databases (UPCVgait, UPCVgaitK2, and SDUgait) and in a straight database that thinks about capricious practices while browsing.

Kin Soo et al., [19] have agreed to gait using cell phones in nature. Unlike the usual techniques for a person to browse a particular street and / or at a normal pace, the proposed technique collects hidden browsing information hidden under uncontrolled conditions without the customer knowing when, where and how. Top-down learning strategies are provided to learn and display the biometrics that can be browsed depending on the information being used, to differentiate the best ones, and to execute the approval process. In particular, the deep neural structure of a compound has been proposed to depict solid phase hierarchies, where highlights in the reserve spaces are continuously summarized by a concise neural system and a fixed neural system. During the investigation, two datasets collected by cell phones for a total of 118 subjects were used for assessments. Experiments show that the proposed strategy executes 93.5\% and 93.7\% accuracy separately on individually identifiable evidence and approval.

Hanking Chao et al., [20] have proposed another point of view that utilizations climbing as an inside and out set, implying that a mobile casing is incorporated by a worldwide neighborhood coordinated profound organization enlivened by our left and right sides of the equator. Cycles data to recognize data that can be utilized in distinguishing proof. In view of this profound set viewpoint, the strategy was resistant to outline changes, and casings could be normally coordinated from various recordings got under various shots, for example from various points, diverse wear or distinctive thing conveying conditions. Examinations show that under ordinary walk conditions, the single-example technique accomplishes a normal positioning 1 precision of $96.1 \%$ in the CASIA-B style data set and $87.9 \%$ in the OU-MVLP style information base. Under different complex conditions, our model likewise shows undeniable degrees of solidarity. It accomplishes $90.8 \%$ and $70.3 \%$ exactness in CASIA-B under sack conveying and coat-wearing strolling conditions, individually, which altogether improves existing prescribed procedures. Besides, the proposed strategy keeps up agreeable exactness regardless of whether just a few casings are accessible in the test models; For instance, it comes to $85.0 \%$ of CASIA-B in any event, when utilizing just 7 edges.

\section{Proposed System Model}


To identify the people, the recognition part is essential and important operation. The identification of human with the consideration of biometric is an efficient way because it connected with the specific information and it easy to transfer the information to anywhere. Normally, the biometrics is a behavioral or physiological characteristic that can be utilized to verify and identify the peoples based on their individuality. They are physiological such hand scans, iris scans, face recognition and fingerprints. The speech recognition and keystroke scan also considerable. The gait recognition is a new biometric identification method which objective to identify the people at very close or large distance based on their walking style. Normally, the gait recognition technique utilizes silhouette images which defined a person in every frame. Moreover, silhouette images can reduce the fine-grained spatial data. Additionally, most research paper did not consider complex scenario of silhouette images. Based on the visual clues, the peoples are recognized in silhouette images not considered gait features. Hence, the conventional methods not suitable to utilize for identify the humans based on their behavior. In this paper, ADBNN-BWO is developed to identify the human from the images. The complete architecture of the proposed system is illustrated in figure 1.

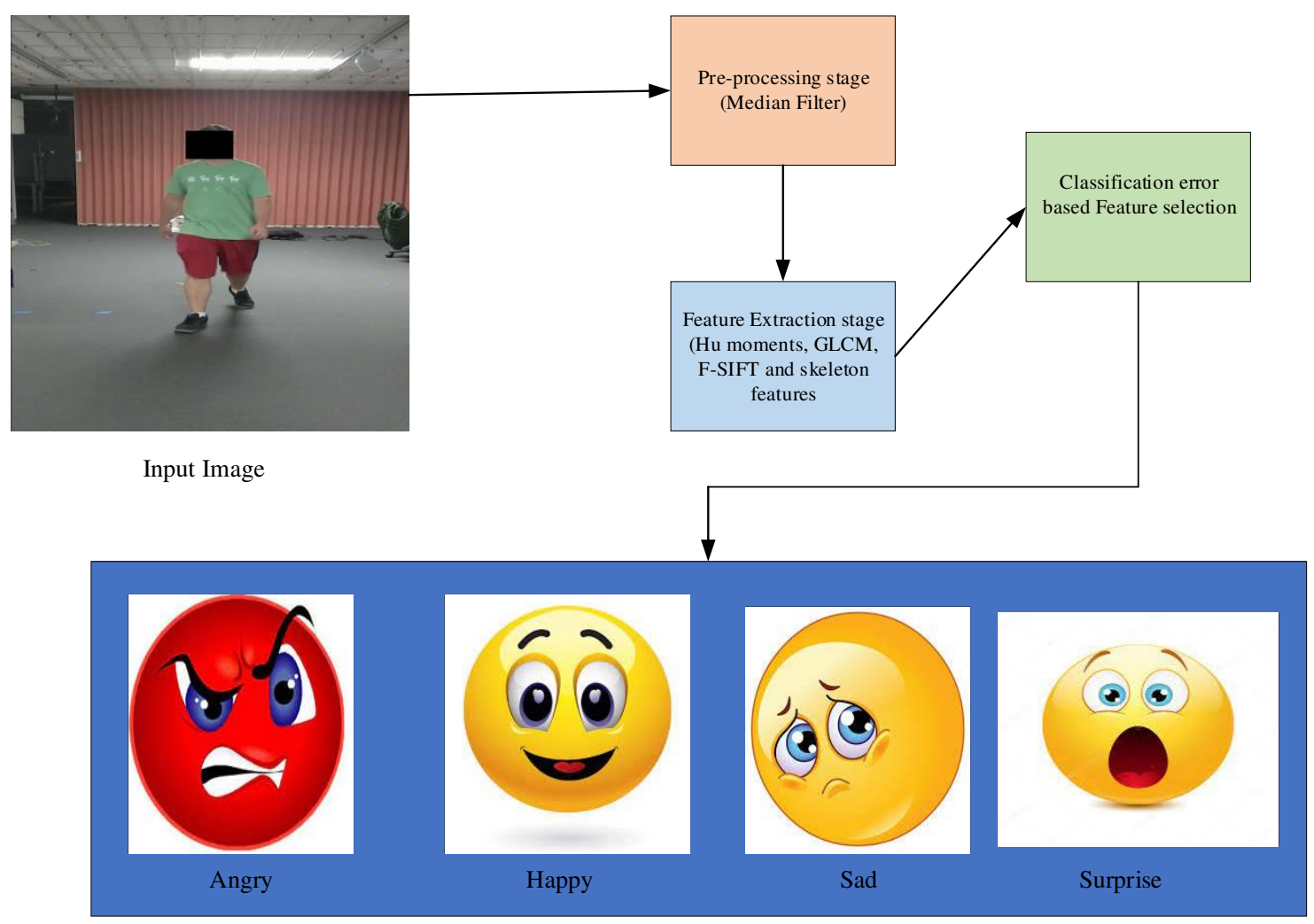

Classification Stage- (DBNN-BWO) Four classes

Figure. 1 System Architecture for gait recognition 
In this proposed methodology, the proposed method is working with four steps such as pre-processing, feature extraction, feature selection and classification. In the pre-processing step, contrast enhancement is achieved with the help of median filter. In the feature extraction step, the Hu Moments, GLCM and F-SIFT and skeleton features are utilized. In the feature selection step, the mean operation is utilized to select essential features among the features. Finally, the ADBNN-BWO is utilized to identify the humans by walking style. The detail description of the proposed steps is presented in below section.

\subsection{Pre-Processing Step}

The pre-processing stage is an essential to remove unwanted noise presented in collected images. In the proposed methodology, the median filter is utilized. the median filter replaces the pixel parameter in the image through the median of the filtering window. In the median filter, the closest pixel values are considered [21]. The mathematical formulation of the median filter is presented in below,

$$
\begin{aligned}
& m f=\operatorname{Median}(P A)=\frac{\sum_{I=1}^{N} P^{I}(N+1)}{2} \text { if } N \text { is odd } \\
& m f=\operatorname{Median}(P A)=\sum_{I=1}^{N} P^{I}\left(\frac{N}{2}\right)+1, \text { if } N \text { is even }
\end{aligned}
$$

Where, the pixel values can be denoted as $P^{I}$. This median filter is changing the central or median pixel of the processing window. The processing pixel can be 0,255 or different digital among 0 and 255 . This pixel exchange can be proceeding with equally in the median filter. Based on this method, the unwanted noises are removed by exchanging the pixel values.

\subsection{Feature Extraction Step}

Once complete pre-processing stage, the feature extraction is considered to extract essential features from the input images. In this proposed methodology, four types of features are considered such as Hu moments, GLCM, F-SIFT and skeleton features.

\subsubsection{Hu moments}

The hu moments also utilized to extract the features from the image. the Hu moments use the central moments where it can be generally the similar as the moments just expressed except that the values of $Y$ and $X$ in the formulations which replaces through the mean values. The hu moments are mathematically presented as follows,

$$
\mu^{P, Q}=\sum_{I=0}^{N} I(X, Y)\left(X-X^{a v g}\right)^{P}\left(Y-Y^{a v g}\right)^{Q}
$$

Where, 


$$
\begin{gathered}
Y^{a v g}=\frac{M 01}{M 00} \\
X^{a v g}=\frac{M 10}{M 00}
\end{gathered}
$$

The main objective of that, by mixing the various normalized central moments which is possible to generate invariant functions mentioning various characteristics of the images.

\subsubsection{GLCM}

In this proposed methodology, the texture-based feature extraction method is selected to extract the essential features from the images such as GLCM. This feature extraction method is initially developed by Haralick and which is most important feature extraction method to extracts the texture features from the images [22]. The GLCM feature behavior second order statistic of image through computing how often pairs of pixels with specified spatial relationship presented in an image. The GLCM matrix is consists of conditional joint probabilities of complete pair wise combinations of gray levels presented in particular distance and displacement distance. The displacement distance can be defined as inter pixel distance. The probability condition of GLCM is presented below,

$$
P^{R}(X)=\left\{C^{I J} \mid(D, \theta)\right\}
$$

Where, displacement distance can be denotes as $(D)$, particular orientation $(\theta)$ and $C^{I J}$ can be represented as gray scale occurrence which formulated as follows,

$$
C^{I J}=\frac{P^{I J}}{\sum_{I, J=1}^{G} P^{I J}}
$$

Where, $P^{I J}$ can be described as probability value of GLCM parameters, $G$ can be represented as number of gray levels.

The features are created with the computation of features for every one of the co-occurrence matrices achieved by the way of $135^{\circ}, 90^{\circ}, 45^{\circ}$ and $0^{\circ}$. After that, the four values are averages. Based on GLCM features, the different texture features are extracted such as Homogeneity, energy, correlation and contrast. In this proposed methodology, these four features are considered from the second order statistics. Additionally, the displacement distance and orientation process are presented below,

$$
\begin{aligned}
& \text { Kernel }=[01 ; 02 ; 03 ; 04 ;, \ldots-11 ;-22 ;-33 ;-44 ; \ldots-10 ;-20 ;-30 ;-40 ; \ldots-1-1 ;-2-2 ;-3- \\
& 3 ;-4-4
\end{aligned}
$$

Based on kernel function, the GLCM considered four displacement distances $\mathrm{D}=1,2,3,4$ and for angles, orientations. From the consideration of GLCM, the essential features are computed. 


\subsubsection{F-SIFT}

The F-SIFT feature extraction contains the four process such as local image descriptor, orientation assignment, optimal key point localization and scale space extrema detection. Initially, the scale space extrema detection which identified the key point candidates [23]. The initial step is convolute the images with gaussian filter which presented below,

$$
S^{K}=S(K, Y, K \sigma)=g f(X, Y, k \sigma) * S^{K-L}
$$

Where, $S^{0}=I(X, Y), S^{K-1}=I(X, Y), K=1$ which is input image and, gaussian filter is formulated as follows,

$$
g f(X, Y, k \sigma)=\left(\frac{1}{2} \pi(k \sigma)^{2}\right) e^{-\frac{\left(X^{2}+Y^{2}\right)}{2 \pi(k \sigma)^{2}}}
$$

After that, the key points are localized by avoiding some low-key points in the key point localization with edge detection test and low contrast. The low contrast test avoids the unstable extrema location which presented as,

$$
X^{\prime}=-\left(\frac{\partial^{2} D O G^{-1}}{\partial X^{2}}\right)\left(\frac{\partial D O G}{\partial X}\right)
$$

Based on the F-SIFT, the features are extracted from the images. The extracted images are utilized to classify the human walking style from the image database.

\subsubsection{Skeleton Features}

The skeleton feature is an essential feature to identify the human walking style from the images. The local features are extracted from the skeleton joints of the images [24]. The skeleton features are divided into two types regarding to their physical interpretation. The Skelton features of the specific body parts are presented below,

$$
V_{I}^{S}=\frac{P_{I}^{S+1}-P_{I}^{S-1}}{\Delta t} \mid 1<S<\tau
$$

Where, $S$ can be represented as skeletons $S=\{1,2, \ldots, \tau) . \tau$ can be represented as number of skeletons in the images, $\Delta t$ can be represented as interval among two different sequences $S-1$ and $S+1, P_{I}^{S}$ can be represented as coordinate of joint. The complete skeletons of the images are presented in equation (9). From the skeleton features, the 15 joint features are computed such as left foot, left knee, left hip, right foot, right knee, right hip, spine, left hand, left elbow, left shoulder, neck, head, right shoulder, right elbow and right hand.

\subsection{Feature Selection Step}

From the consideration of the feature extraction method, the various kinds of features are computed. Among them, the essential features are selected to identify the human walking style-based emotions. The proposed method 
uses a fitness function which involves the number of features and classification error rate. The mathematical formulation of the fitness function is presented follows,

$$
F i t=\alpha E R(k)+\beta \frac{|F S|}{|D|}
$$

Where, $E R$ can be described as classification error rate, $D$ can be described as complete feature sets from the database, $|F S|$ can be described as selected feature set and $\alpha, \beta$ can be described as reference value such as $0.52,0.99$ respectively. Based on the above formulation, the essential features are selected which enhance the performance of the classification.

\subsection{Classification Step}

The selected features are finally sent to the classification stage. The classification stage is utilized to identify the humans based on their walking style. The proposed approach is utilized to select the emotions from the images. The hybrid ADBNN-BWO is utilized and it is a combination of DBNN and BWO. In the DBNN, the weight parameter is selected with the help of BWO algorithm. The detail description of the DBNN and BWO is presented in below section.

\subsubsection{Deep Belief Neural Network}

Deeper constructions are quicker and more efficient than superficial ones. For example, a few capabilities can be summarized with a deep NN, while a system with a deep 1 requires a large number of computational units and boundaries. Superficial designs may require different components and thus, models are made to handle completely different capabilities. Nevertheless, the test for producing deeply different NNs has been slippery for some time. It develops in a way that is not straight on many levels, based on the impossibility of development techniques that rely on conventional inclinations. This has changed with the advancement of DPNs that have been used effectively for some, including regulation, regeneration [25], scale reduction, division, data recovery, language preparation, machine technology, discourse, sound and hybrid separation. Performance Directions. The structure of DBNs is stabilized by a number of layers that are stacked one on top of the other so that building a system can capture the essential standards and types directly from the basic source information. Each RBM, within a certain layer, receives the contributions of the last layer and feeds them to the next layer, which gradually elevates everything individually, allowing for optimal conditions. Creating a DBN produces each RPM independently, starting at the basic level and progressing bit by bit, with the top layer RPM. The DBN configuration is given in Figure 2. 


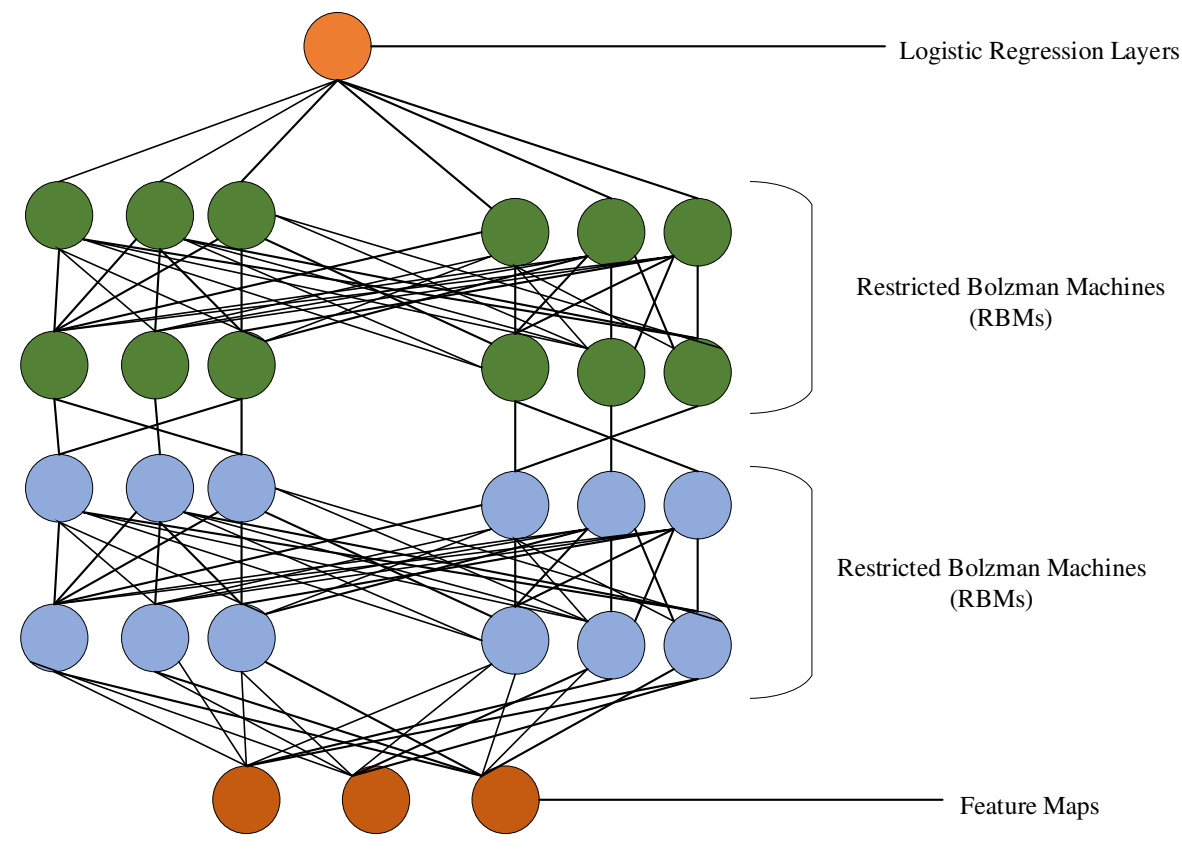

Figure. 2 Structure of Deep Belief Neural Network

An RPM is an energy-based production model in which I have parallel observable units (observed factors), where $\mathrm{v}=[v 1, v 2, \ldots, v I]$ wherevi $\in\{0,1\}$, and $\mathrm{J}$ is a layer Includes secret units (explanatory elements), where $h=$ $[h 1, h 2, \ldots, h]]$ whereh $j \in\{0,1\}$, with bilateral weight associations. RBMs follow the encoder-decoder worldview. In this worldview an encoder changes the contribution of an element vector representation from which a decoder can recreate the first information. In the case of RBMs, both coded imagery and (decoded) remaking are naturally normal. The encoder-encoder design is involved in shedding light on: (i) Following the production, the element vector can be processed in an auxiliary way and (ii) the model can analyze how well it captures significant data by recreating the information. Given the observed express, the energy of the combined design of the transparent and closed units $(v, h)$ is provided by the condition underlying,

$$
\begin{aligned}
& E(v, h)=-C V^{T}-b h^{T}-h W v^{T} \\
& =-\sum_{i=1}^{I} C_{i} V_{i}-\sum_{j=1}^{j} b_{j} h_{j}-\sum_{j=1}^{J} \sum_{i=1}^{I} W_{j i} v_{i} h_{j}
\end{aligned}
$$

Where $W \in I R^{J \times I}$ is a lathe with $\mathrm{RBM}$ association loads, $c=[c 1, c 2, \ldots, c I] \in I R^{I}$ is the forerunner of observable units and $b=[b 1, b 2, \ldots, b J] \in I R^{J}$ Inclination of secret units. To break the equilibrium, loads are usually introduced with small irregular properties (for example somewhere in the range of .00.01 and 0.01). The secret slope can introduce $b_{j}$ with an enormous negative value (e.g. -4$)$ to enhance porosity and significant units' dependence, $c i$, 
recording to $\log \left(\frac{p_{i}}{1-p_{i}}\right)$, where $p_{i}$ is the number of vectors produced in which vi $=1$. The learning method needs to be changed (in early productions) given that the noticeable unit is likely to have been activated i, so gradually merges with $p_{i}$. RBM expresses an opportunity for each system $(v, h)$, which is depicted in the underlying condition,

$$
p(v, h)=\frac{e^{-E(v, h)}}{Z}
$$

Where $Z$ is a standardization constant called parcel work in solidarity with the actual structure, which is obtained by summarizing the energy of all imaginary $(v, h)$ systems.

$$
z=\sum_{v, h} e^{-E(v, h)}
$$

Since there is no correlation between any two units within the same layer, given that a certain irregular information configuration is $\mathrm{v}$, each secret unit is free from each other, and $h$ is the probability of a given $v$,

$$
p(h \mid v)=\prod_{j} p\left(h_{j}=1 \mid v\right)
$$

Where,

$$
p\left(h_{j}=1 \mid v\right)=\sigma\left(b_{j}+\sum_{i=1}^{I} v_{i} w_{j i}\right)
$$

For execution purposes, $h_{j}$ is set to 1 when $p\left(h_{j}=1 \mid v\right)$ is more important than the given random number (assigned somewhere in the range 0 and 1 ) and in any case 0 . In addition to a certain secret level, the probability of $h$ given $v$ is given,

$$
p(v \mid h)=\prod_{i} p\left(v_{i}=1 \mid h\right)
$$

Where,

$$
p\left(v_{i}=1 \mid h\right)=\sigma\left(c_{i}+\sum_{j=1}^{j} h_{j} w_{j i}\right)
$$

When using the information vector to reproduce (18), it is necessary to double the confidentiality levels. The use of real probabilities would really abuse the data barrier, which goes into a rigid order and is forced to bypass everything that treats secret units as little data. Given the external potential sent to the apparent vector, $V$,

$$
p(v)=\sum_{h} p(v, h)=\frac{1}{z} \sum_{h} e^{-E(v, h)}
$$

Then, if a specific product vector is given, its change can be elevated by changing $t$.

\subsubsection{Process of Black Widow Optimization}

The proposed algorithm of BWO is utilized to finding the optimal weighting parameter for achieving identification of the images. The classification of human moments is identified from the images which are also checked 
in the proposed algorithm. The proposed BWO algorithm is a process of BWO algorithm. In this section, a detailed description of, BWO, and the proposed algorithm are presented.

\subsubsection{Black widow optimization}

This algorithm can be characterized through the unique mating characteristics of black widow spiders. In the BWO algorithm, the main stage of the exclusive stage which named cannibalism. Spiders can be considered as airbreathing arthropods that contain eight legs also with venomous fangs. From the different types of organisms, these species are the arachnids the larger order which rank seventh incomplete species diversity. From November 2015, taxonomists can record randomly 114 families with 4700 spider species. Moreover, dimension has been increased within experts as to how complete these relations can be divided [26]. From the different scenario, more than 20 different taxonomies which have been considered from 1900. The BWO algorithm starts with an initial spider population and every spider describes a result. These initial spiders, in pairs which attempt to generate the new generation. In the BWO algorithm, the female black widow noshes the male throughout and afterward mating. After that, the female black widow stowed sperms in her sperm in addition produce the egg in sacks. After 11 days, it is coming with egg sacs. The basic characteristics of BWO are presented as follows,

\section{Initial population}

In the BWO algorithm, the possible answers to every issue have been measured as a black widow spider. Every black widow spider is considered as issue parameter. The initial population of BWO is formulated as follows,

$$
W=\left[X^{1}, X^{2}, \ldots, X^{N^{\text {Var }}}\right]
$$

Where, $N^{\text {Var }}$ can be described as a dimension of parameters, $X^{1}, X^{2}$ can be considered as the floating-point number achieved with the fitness function. The fitness function is formulated as follows,

$$
F(W)=f\left[X^{1}, X^{2}, \ldots, X^{N^{\text {Var }}}\right]
$$

To imitate the optimization algorithm, the candidate widow matrix is created with the early populace of spiders. After that, pairs of parentages were arbitrarily designated to operate the breeding process through breeding which the male black widow is plagued through the female before and after.

Once the initial population is completed, the fitness function is computed. Based on the fitness function, the optimal weighting parameter of DBNN. The fitness function is evaluated with the consideration of the PSNR value. The PSNR should be maximized to enable efficient secure operation. Hence, the fitness function is formulated with 
the maximization of PSNR. The fitness function is achieved by selecting the optimal weighting parameter value. The fitness function is mathematically formulated as follows,

$$
\begin{aligned}
& \mathrm{FF}=\text { MAX\{PSNR }\} \\
& P S N R=10 \log _{10}\left(\frac{M A X^{P}}{M S E}\right) \\
& M S E=\frac{1}{N * M} \sum_{X=1}^{N} \sum_{Y=1}^{M}\left[I_{\text {image }}(A, B)-I_{d-\text { image }}(A, B)\right]^{2}
\end{aligned}
$$

Where, $I_{d \text {-image }}(A, B)$ is described as identified images and $I_{\text {image }}(A, B)$ is described as an input image. Based on the fitness function, the weight parameters are selected which are utilized to enhance the optimal classification procedure.

\section{Procreate}

In the BWO algorithm, the pairs are independent which initiates to mate to generate the new generation as well as mate with separately. In real-world applications, more than 1000 eggs are generated in every mating process. At finally, the spider babies are growing and very strong. So, it is generated with an extended as widow array with chance generation. Based on descendant's operation, the procreate is formulated as follows,

$$
\left\{\begin{array}{l}
Y^{1}=\alpha \times X^{1}+(1-\alpha) \times X^{2} \\
Y^{2}=\alpha \times X^{2}+(1-\alpha) \times X^{1}
\end{array}\right.
$$

This procedure can be repeated at different times by randomly chosen numbers that may not be duplicated. At last, the array is sorted based on fitness parameters and cannibalism rating. Additionally, the optimal solutions are collected to the afresh created population.

\section{Cannibalism}

In the BWO algorithm, the three different types of cannibalism are presented. The initial one is named sensual cannibalism that process of a female widow chucks her husband before or after breeding. Based on the female and male cannibalism, the fitness values are evaluated.

\section{Mutation}

In the mutation process, the mute pop number is automatically selected with the population. Each solution can be selected randomly transfers two elements in the array structure. The mutation rate is computed based on mute pop.

\section{Convergence}


Similar to other algorithms, the termination condition can be checked with three steps such as (1) reaching the specified level of accuracy, (2) adherence of no variation in the fitness value, and (3) several iterations. The BWO is practical in some benchmark optimization issues, the optimal solutions can be collected. The termination condition was also checked [27].

The adaptive projected technique is utilized to select the optimal weight parameters of the projected encryption algorithm. The BWO optimization algorithm is enhanced with the help of the fitness function to empower the classification by selecting the optimal weighting parameter. The complete flowchart of the proposed method is illustrated in figure 3 .

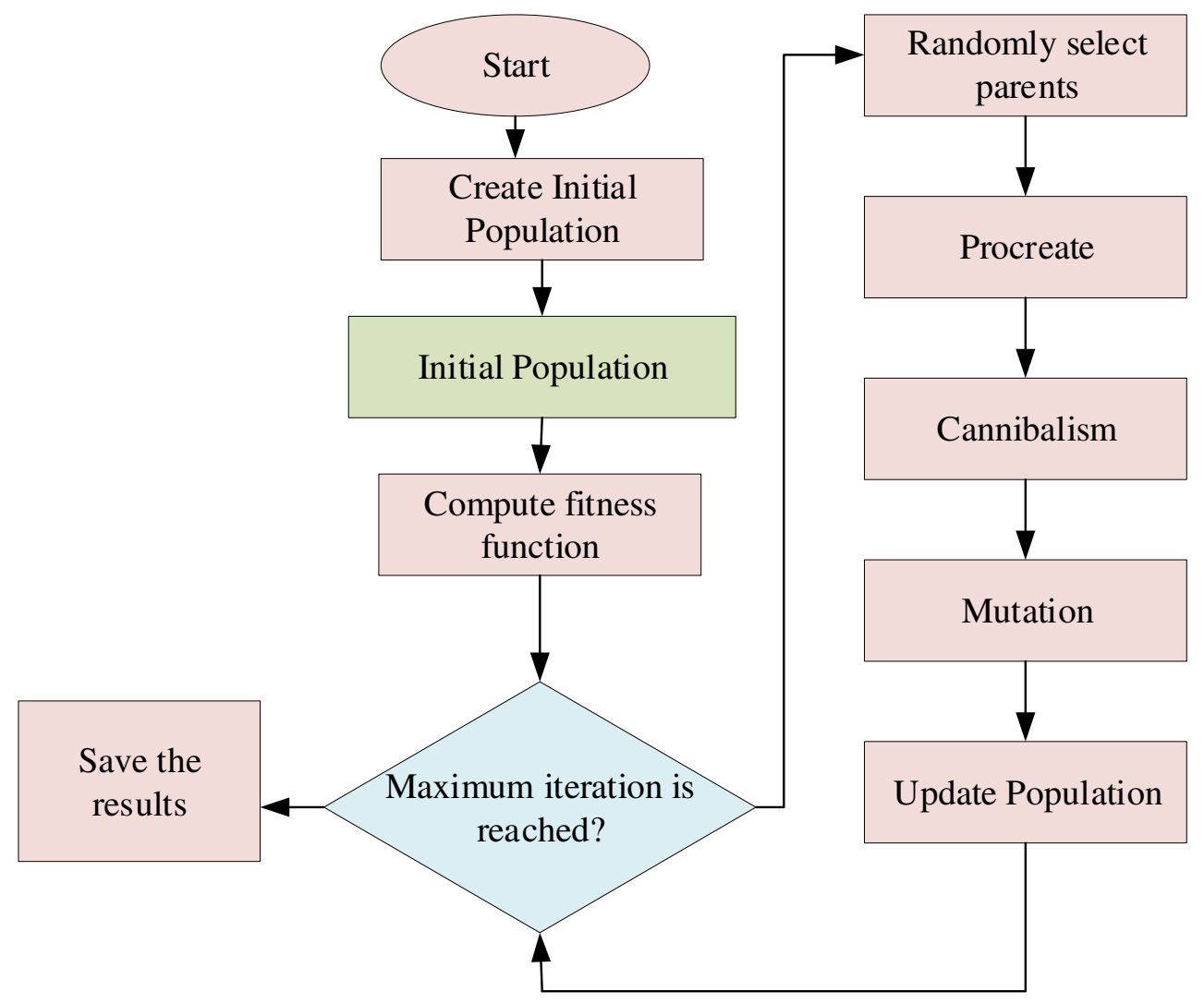

Figure. 3 Flowchart of the BWO

Initially, random weighting parameters are selected in the DBNN which degrades the classification parameter. The optimal weighting parameter selection is an essential task to enable an efficient classification. The initialization of the algorithm is a random weighting parameter of the classification. The optimal weighting parameters of DBNN are selected with the consideration of the BWO algorithm. The BWO is utilized to select the optimal 
weighting parameters with reverse solution initialization. Finally, the proposed algorithm is utilized to select the optimal weighting parameters of the proposed methodology.

\section{Performance Evaluation}

The performance of the proposed methodology is evaluated and justified in this section. The proposed methodology is validated with the consideration of statistical measurements such as accuracy, precision, recall, sensitivity, specificity and F_Measure respectively. The proposed methodology is compared with existing methods such as MA-PSO and PSO-RNN respectively. The proposed method can be implemented with the consideration of collected database [28]. From the database, collected videos are considered to individual walking style. The dataset consists of emotion labels with four different emotions such as Happy, angry, sad and surprise. These gait videos are extracted from RGB videos or motion captured. The implementation parameters of the proposed method are presented in table 1 . The proposed method is validated by statistical measurements. The performance metrices of the statistical measurements are presented below in this section. The sample databases are illustrated in figure 4.

Table. 1 Implementation parameters of the proposed methodology

\begin{tabular}{|c|c|c|c|}
\hline S. No & Method & Description & Value \\
\hline 1 & \multirow{9}{*}{ Proposed method } & Minimum batch size & 500 \\
\hline 2 & & Max Epochs & 15 \\
\hline 3 & & Initial learn rate & 0.05 \\
\hline 4 & & Learn rate drop factor & 0.2 \\
\hline 5 & & Learn rate drop period & 5 \\
\hline 6 & & Momentum & 0.9 \\
\hline 7 & & Initial population & 50 \\
\hline 8 & & Maximum iteration & 100 \\
\hline 9 & & Inertia factor & 0.7298 \\
\hline 10 & \multirow{5}{*}{ PSO } & Swarm size & 20 \\
\hline 11 & & Inertia weight & 0.729 \\
\hline 12 & & Positive constant & 1.49445 \\
\hline 13 & & Positive constant & 1.49445 \\
\hline 14 & & Random numbers & $0.8,0.2$ \\
\hline
\end{tabular}

The proposed classifier is used to detect human walking style-based emotion from the images. The human walking style-based emotion are presented during testing process; then human walking style-based emotion is detected. If the human walking style-based emotion is not presented during testing process, then human walking stylebased emotion is not detected. The confusion matrix is computed based on following constraints, 
* A identify human walking style is actually presented and detected as presented scenario which named as True Positive (TP).

* A identify human walking style are actually not presented and detected as not presented which named as True Negative (TN).

* A identify human walking style are actually not presented but detected which named as False Positive (FP).

* A identify human walking style are presented and detected as not presented which named as False Negative (FN).

Based on the progress of confusion matrix terms, the proposed methodology is evaluated by performance metrices which are formulated as follows,

Accuracy: It is defined as number of correctly detected data instances from the total number of instances.

The formula of accuracy is presented follows,

$$
\text { Accuracy }=\frac{T N+T P}{T N+F P+T P+F N}
$$

Precision: It is defined as fraction of positive detected which actually correct. The precision effectively computed a complete probability and performance measure. The recall formulation presented follows,

$$
\text { Precision }=\frac{T P}{T P+F P}
$$

Recall: It can be defined as ratio of correctly detected positive samples to total positive instances which formulated as follows,

$$
\text { Recall }=\frac{T P}{(T P+F N)}
$$

Specificity: It can be defined as ratio of correctly detected negative instances to total negative instances which formulated as follows,

$$
\text { specificity }=\frac{T N}{(T N+F P)}
$$

F_Measure: The F_Measure must be presented among 0 to 1 . The worst value is 0 and best value is 1 . The F_Measure can be represented as follows,

$$
F_{\text {Measure }}=\frac{2 T P}{(2 T P+F P+F N)}
$$




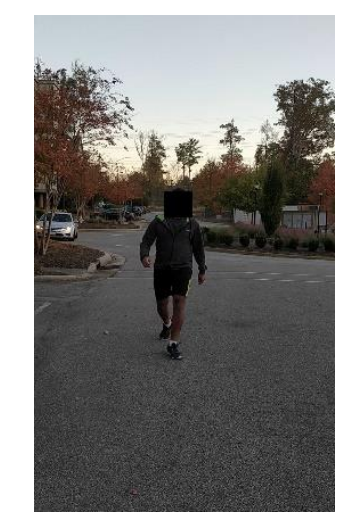

(a)

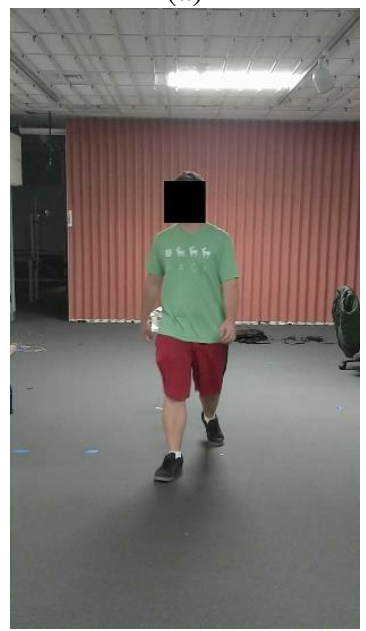

(c)

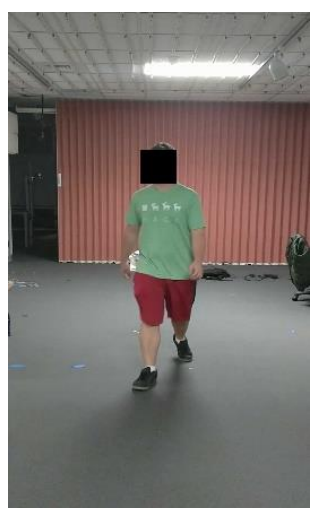

(b)

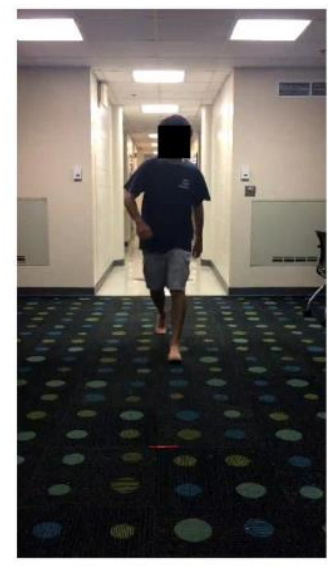

(d)

Figure. 4 Sample images of gait recognition (a) Angry, (b) Happy, (c) sad and (d) surprise

The sample databases are utilized to evaluate the performance of the proposed methodology. The images are sent to the proposed methodology to detect the human walking style-based emotion. The proposed classifier is designed with three existing methods which is compared with the existing design methods of ANN, ANFIS, and MAPSO respectively in terms of statistical measurements. The comparison analysis of the proposed classifier is presented in table 2.

Table. 2 Comparison analysis part

\begin{tabular}{|c|c|c|c|c|c|c|c|}
\hline Method & Class & Features & Accuracy & Precision & Recall & Specificity & F_Measure \\
\hline \multirow{4}{*}{ ANN } & 1 & \multirow{4}{*}{1000} & 0.92 & 0.90 & 0.91 & 0.92 & 0.92 \\
\hline & 2 & & 0.89 & 0.91 & 0.85 & 0.91 & 0.95 \\
\hline & 3 & & 0.87 & 0.86 & 0.91 & 0.87 & 0.92 \\
\hline & 4 & & 0.91 & 0.84 & 0.92 & 0.89 & 0.82 \\
\hline \multirow{5}{*}{ ANFIS } & 1 & \multirow{4}{*}{1000} & 0.93 & 0.91 & 0.89 & 0.87 & 0.89 \\
\hline & 2 & & 0.89 & 0.86 & 0.82 & 0.85 & 0.87 \\
\hline & 3 & & 0.84 & 0.82 & 0.85 & 0.92 & 0.91 \\
\hline & 4 & & 0.92 & 0.90 & 0.92 & 0.89 & 0.89 \\
\hline & 1 & & 0.87 & 0.85 & 0.89 & 0.87 & 0.91 \\
\hline
\end{tabular}




\begin{tabular}{|c|c|c|c|c|c|c|c|}
\hline \multirow{3}{*}{ MA-PSO } & 2 & \multirow{3}{*}{1000} & 0.85 & 0.83 & 0.92 & 0.91 & 0.95 \\
\cline { 2 - 6 } & 3 & & 0.83 & 0.85 & 0.95 & 0.92 & 0.92 \\
\cline { 2 - 6 } & 4 & & 0.91 & 0.89 & 0.98 & 0.89 & 0.93 \\
\hline \multirow{3}{*}{ Proposed method } & 1 & \multirow{3}{*}{1000} & 0.97 & 0.93 & 0.98 & 0.97 & 0.98 \\
\cline { 2 - 6 } & 2 & 0.95 & 0.92 & 0.93 & 0.93 & 0.97 \\
\cline { 2 - 6 } & 3 & & 0.93 & 0.90 & 0.94 & 0.91 & 0.95 \\
\cline { 2 - 6 } & 4 & & 0.96 & 0.93 & 0.96 & 0.95 & 0.91 \\
\hline
\end{tabular}

Class 1: Happy; Class 2: Angry, Class 3: Sad and Class 4: Surprise

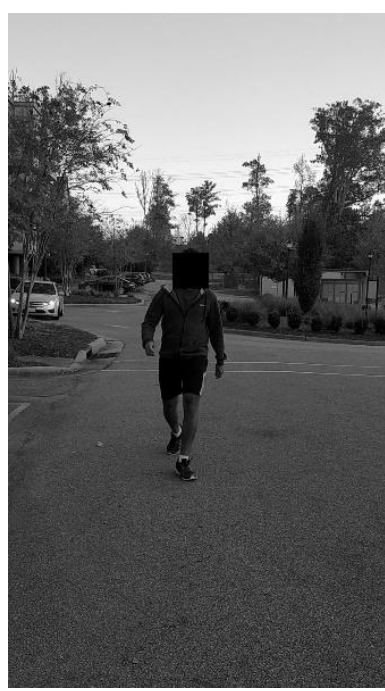

(a)

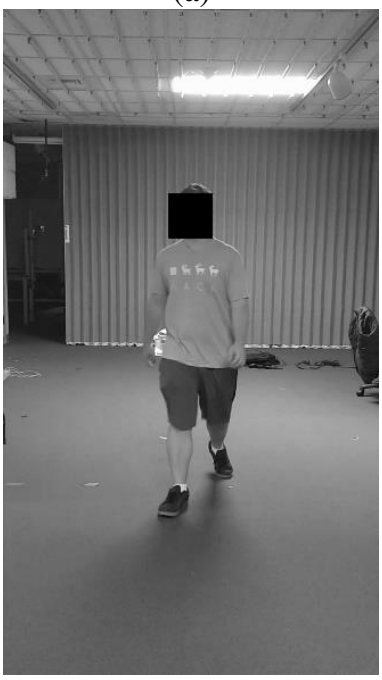

(c)

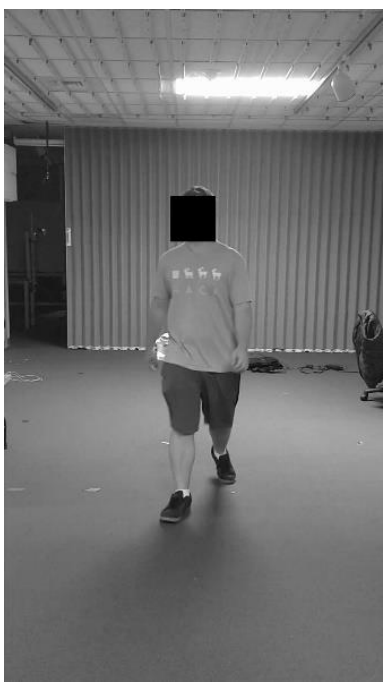

(b)

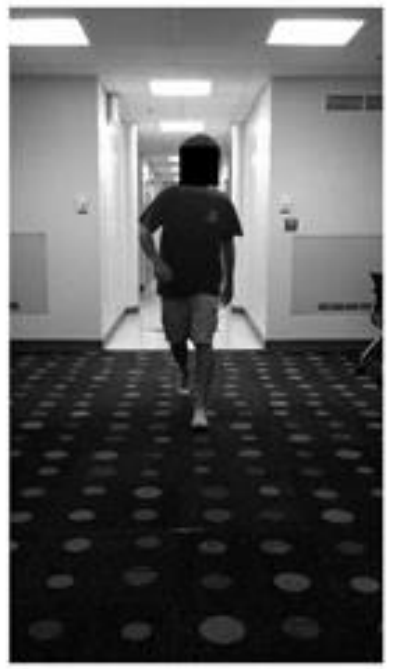

(d)

Figure. 5 Pre-processing images of gait recognition (a) Angry, (b) Happy, (c) sad and (d) surprise 


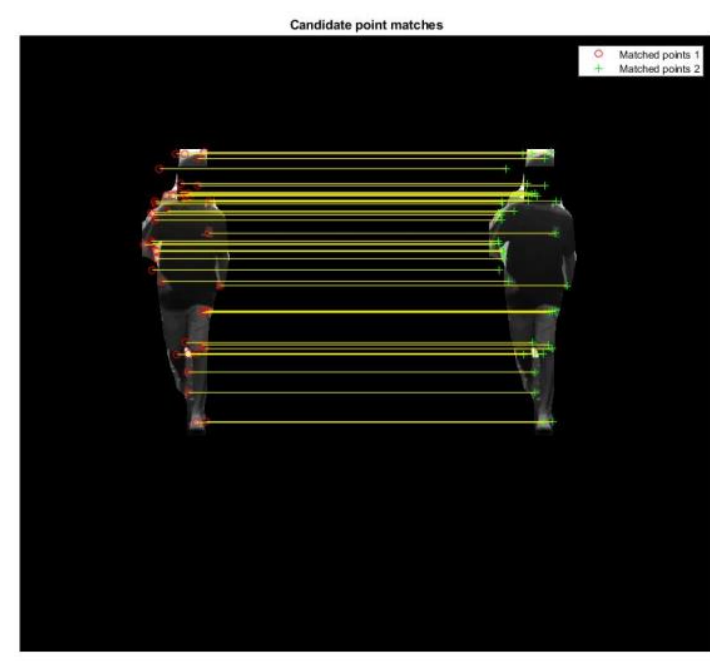

(a)

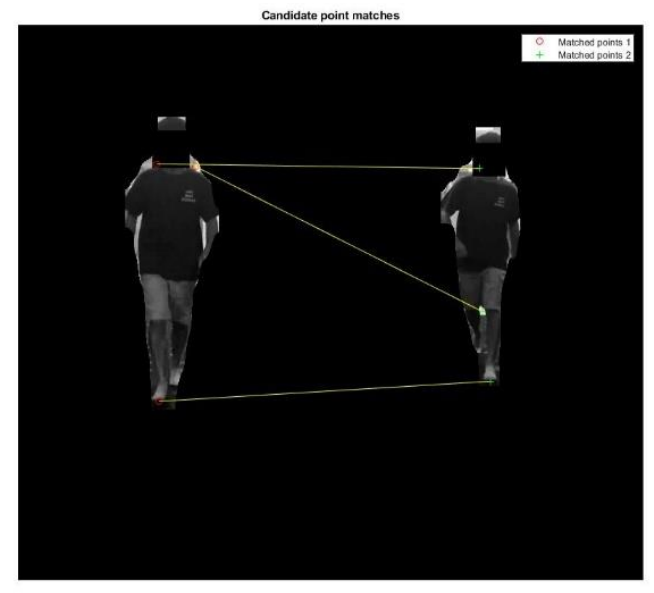

(c)

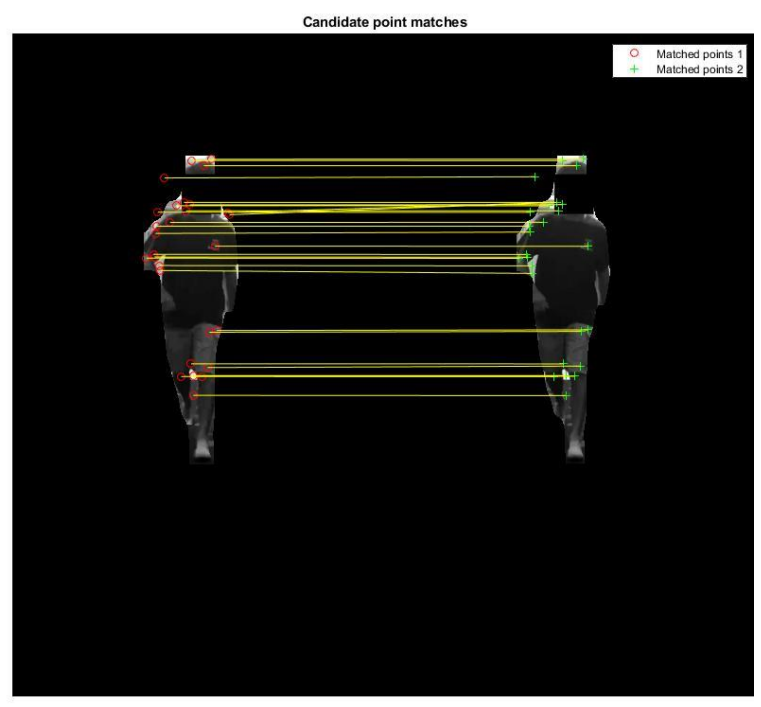

(b)

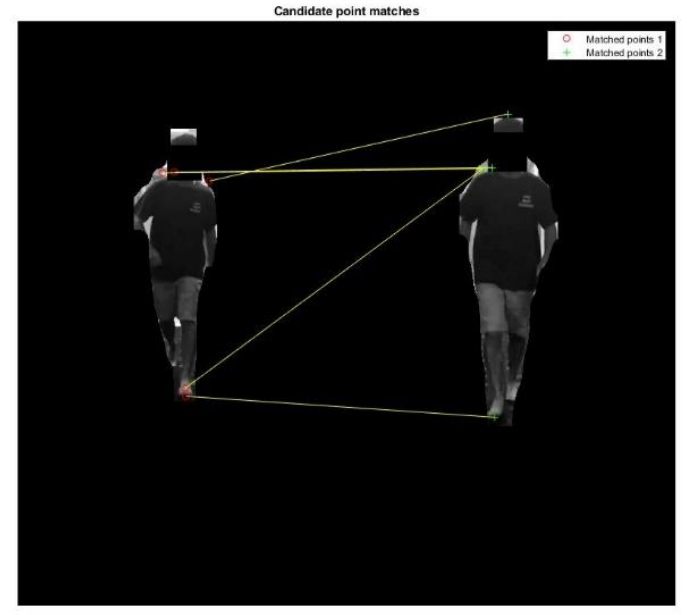

(d)

Figure. 6 Features images of gait recognition (a) Angry, (b) Happy, (c) sad and (d) surprise 


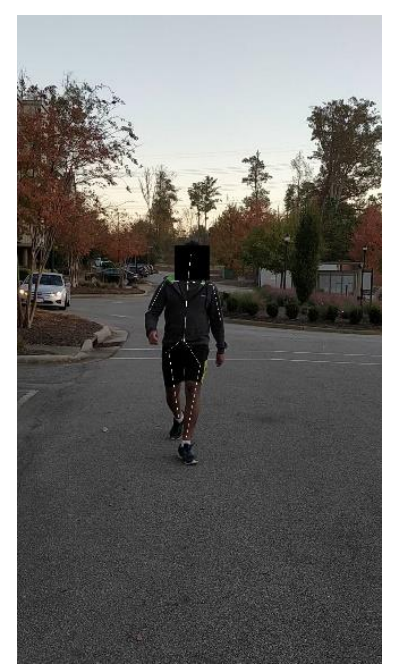

(a)

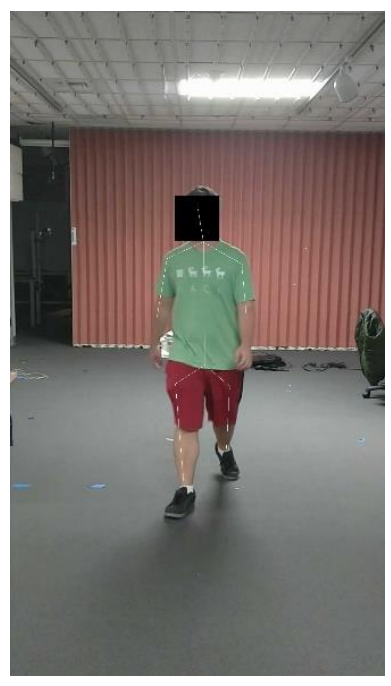

(c)

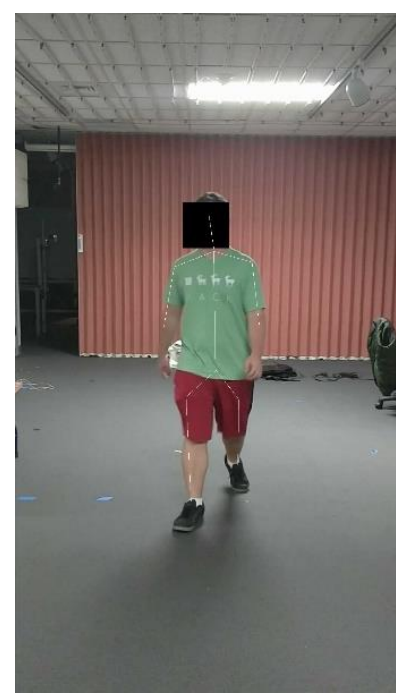

(b)

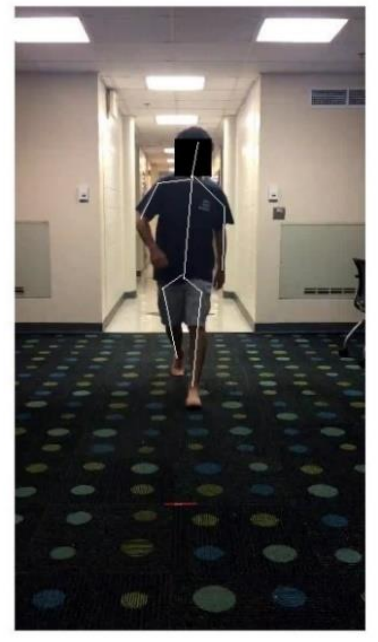

(d)

Figure. 7 Skeleton images of gait recognition (a) Angry, (b) Happy, (c) sad and (d) surprise

The pre-processing image of proposed method is illustrated in figure 5. The feature extraction images of proposed method are illustrated in figure 6. Skeleton images are illustrated in figure 7 . From the table 2 , the proposed method is achieved the best accuracy level in human walking style-based emotion types. The proposed method is achieved the accuracy in four classes which is $0.97,0.95,0.93$ and 0.96 respectively. The ANFIS is achieved the accuracy in four classes which is $0.92,0.89,0.87$ and 0.91 . The ANN is achieved the accuracy in four classes which is $0.93,0.89,0.84$ and 0.91 respectively. Similarly, MA-PSO is achieved the accuracy in four classes which is 0087 , 0.85, 0.83 and 0.91 respectively. From the analysis, the proposed method is achieved the best results more than the existing methods in terms of accuracy. The proposed method is achieved the better values of statistical measurements 
such as specificity, recall, F_Measure, accuracy and precision. The prediction label of each class is presented in table 3.

Table. 3 Prediction level of four classes

\begin{tabular}{|l|l|l|l|l|}
\hline \multicolumn{5}{|c|}{ Predicted Label } \\
\hline Happy & 950 & & & 3 \\
\hline Angry & 1 & 919 & & 3 \\
\hline Sad & 2 & & 919 & 1 \\
\hline Surprise & 4 & & 1 & 901 \\
\hline
\end{tabular}

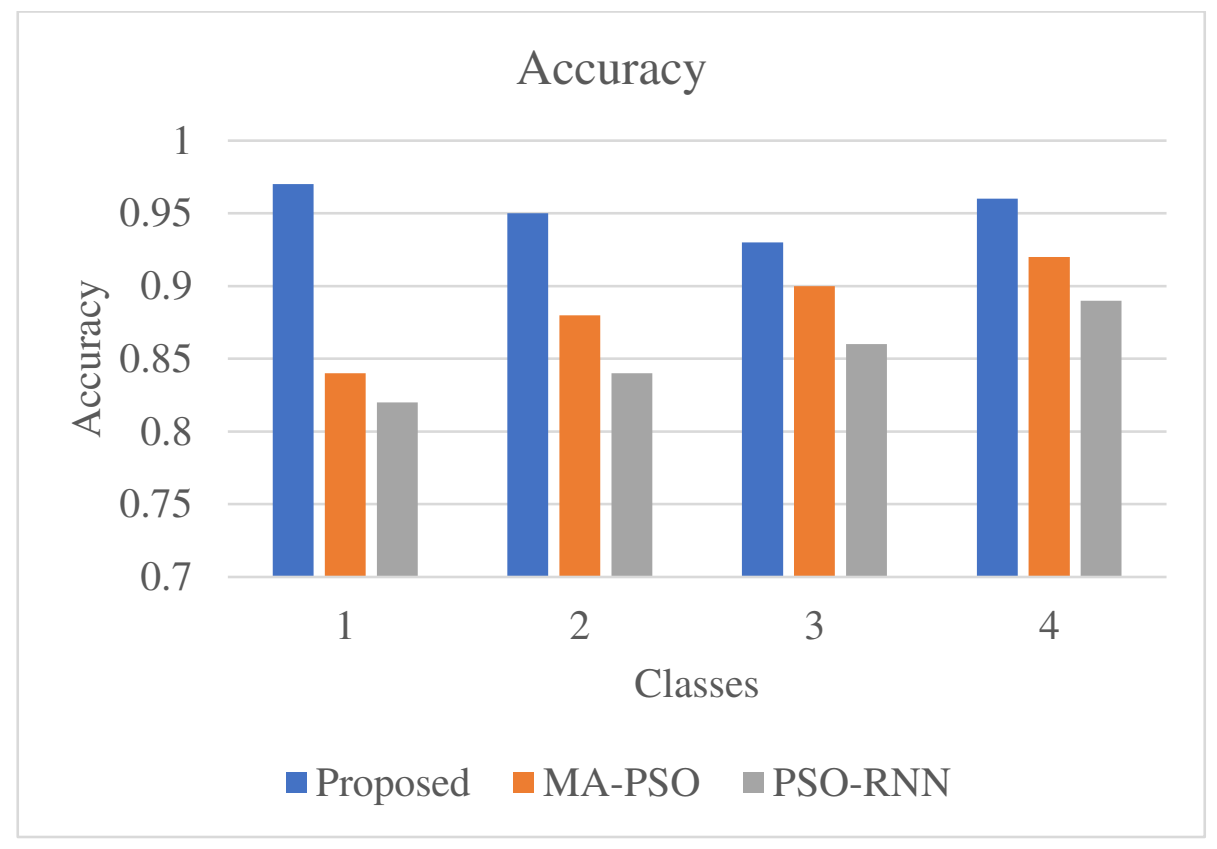

Figure. 8 Analysis of accuracy 


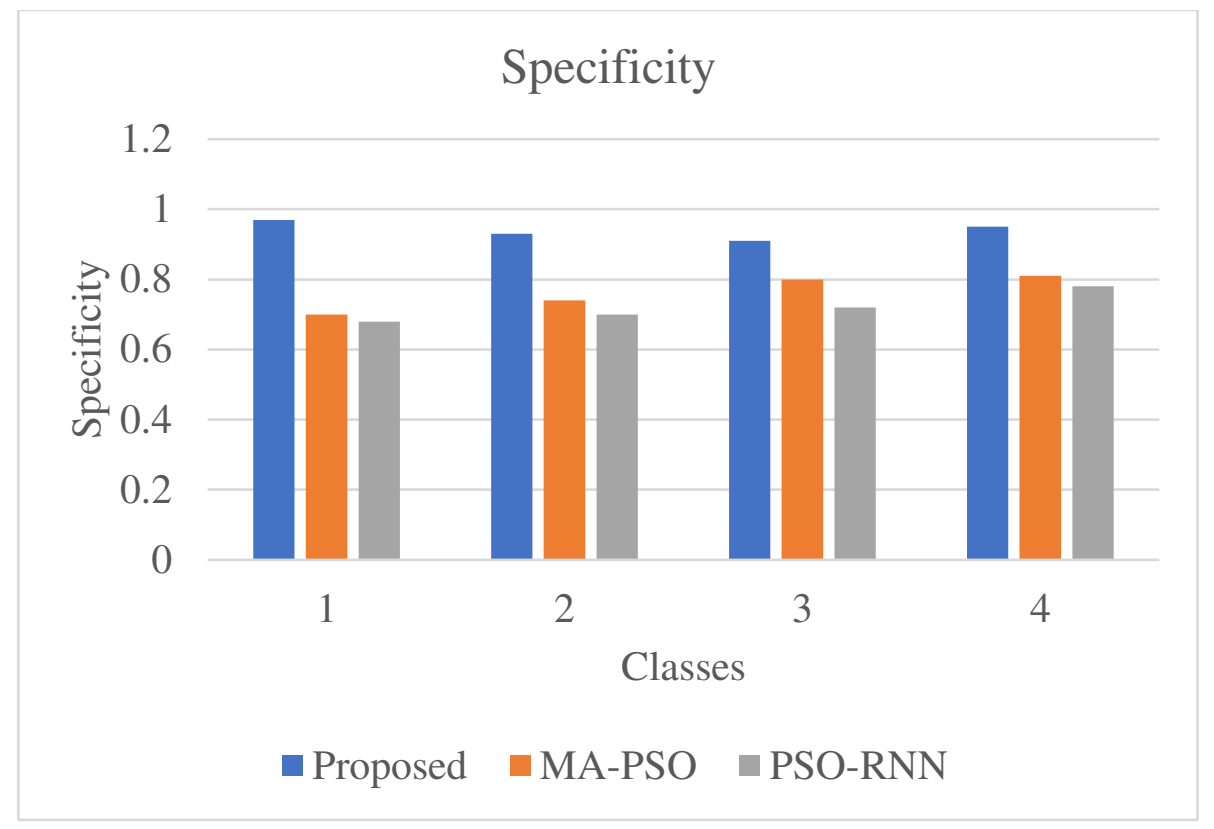

Figure. 9 Analysis of specificity

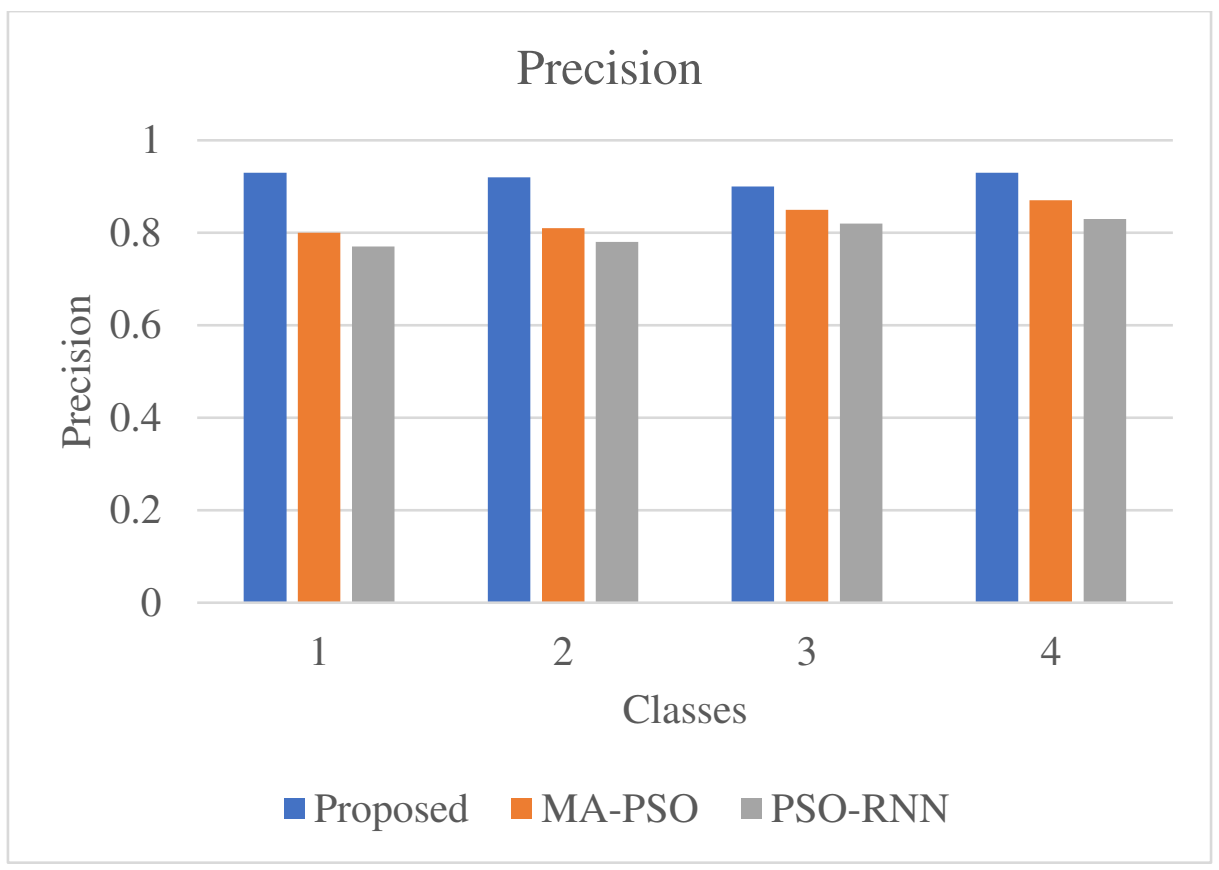

Figure. 10 Analysis of precision 


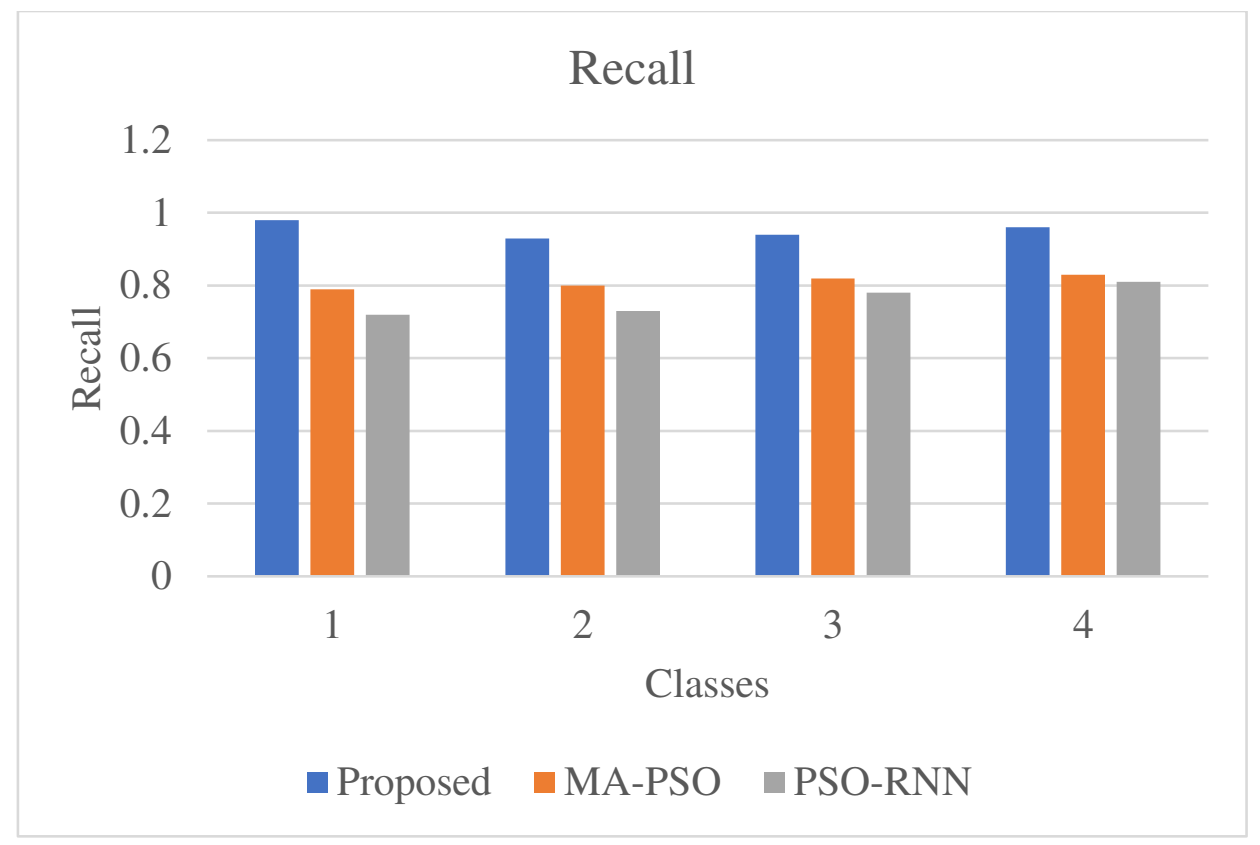

Figure. 11 Analysis of recall

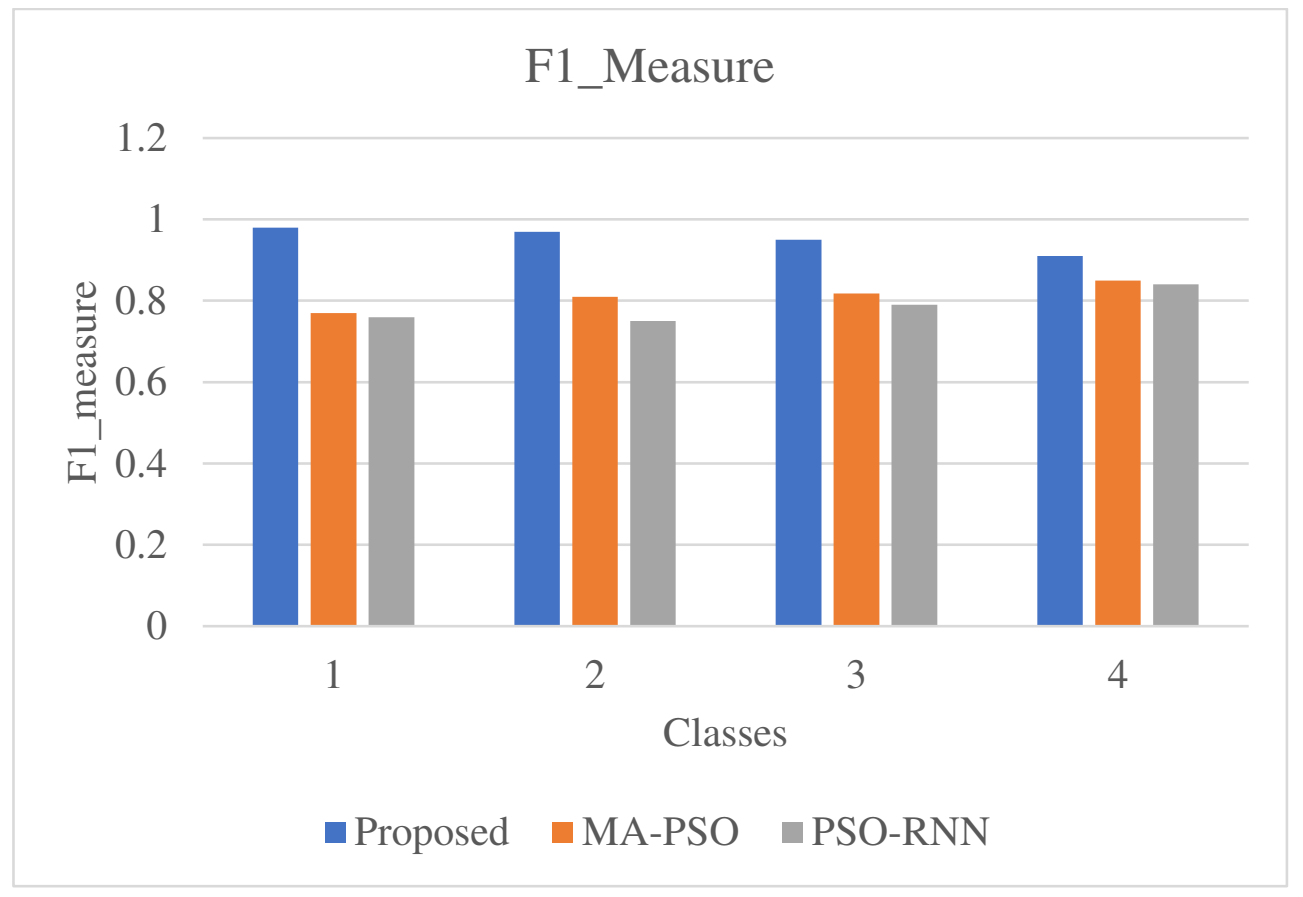

Figure. 12 Analysis of F1_measure 


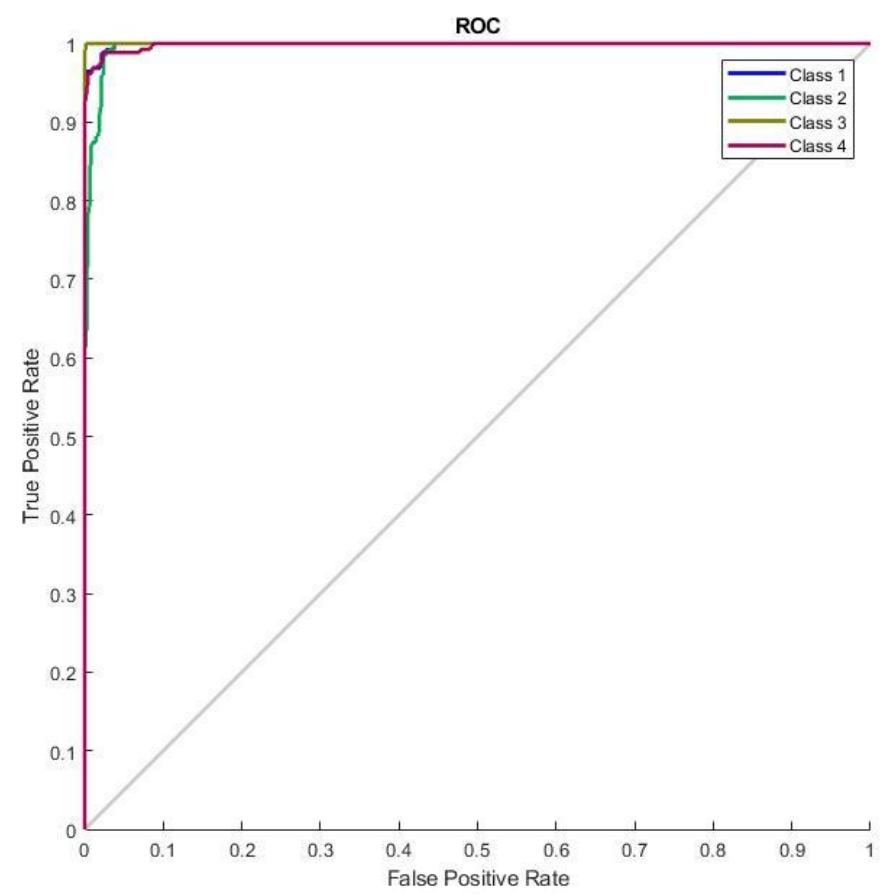

Figure. 13 Analysis of ROC curve

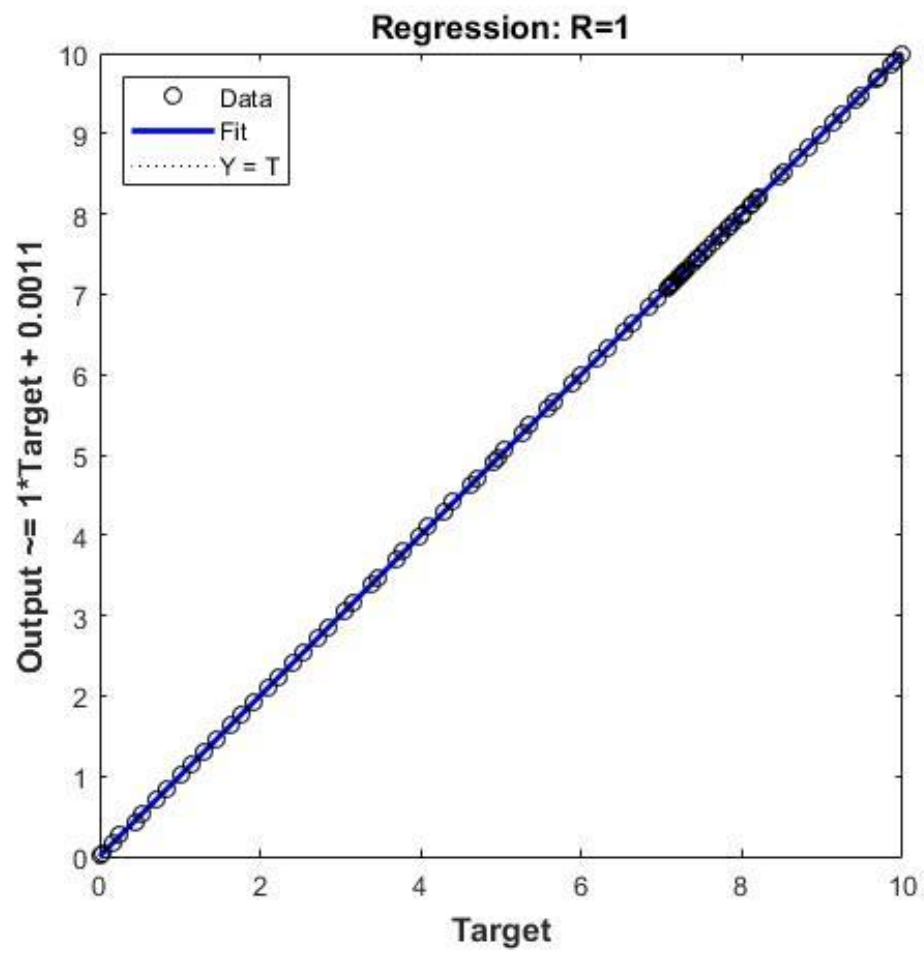

Figure. 14 Analysis of Regression 


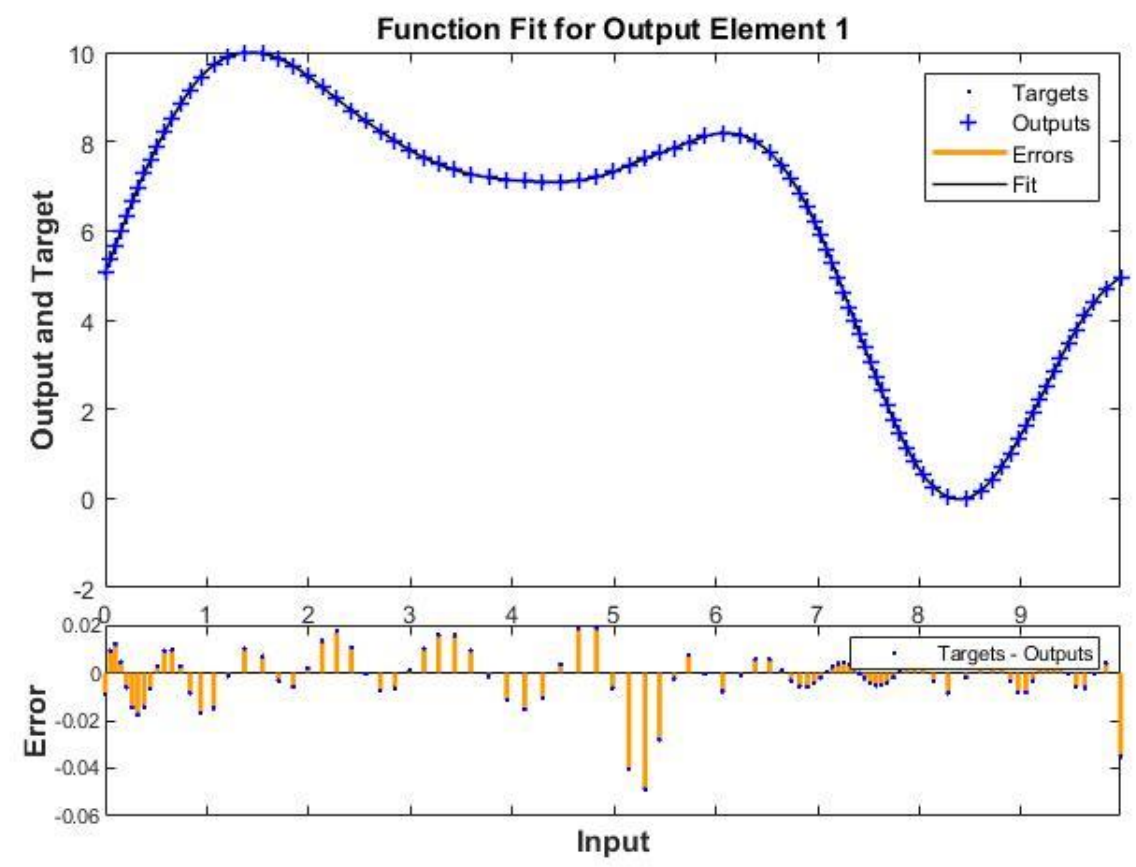

Figure. 15 Analysis of Proposed Error rate

The proposed method is validated by using comparison analysis. In the comparison analysis, the proposed method is compared by MA-PSO and PSO-ANN respectively. The comparison analysis of accuracy is presented in figure 8. From the figure 8, the proposed method is achieved the 0.97 rate accuracy in class 1 . Similarly, the MA-PSO and PSO-RNN methods are achieved the accuracy 0.7 and 0.67 respectively. From the comparison analysis, the proposed method is achieving the best accuracy in every classes in human walking style-based emotion. The comparison analysis of specificity is presented in figure 9. From the figure 9, the proposed method is achieved the 0.97 specificity in class 1 . Similarly, the MA-PSO and PSO-RNN methods are achieved the specificity 0.7 and 0.68 respectively. From the comparison analysis, the proposed method is achieving the best specificity in every classes in human walking style-based emotion. The comparison analysis of precision is presented in figure 10. From the figure 10 , the proposed method is achieved the 0.9 rate precision in class 1 . Similarly, the MA-PSO and PSO-RNN methods are achieved the accuracy 0.8 and 0.77 respectively. From the comparison analysis, the proposed method is achieving the best precision in every classes in human walking style emotion detection. The comparison analysis of recall is presented in figure 11. From the figure 11, the proposed method is achieved the 0.98 rate recall in class 1 . Similarly, the MA-PSO and PSO-RNN methods are achieved the recall 0.79 and 0.72 respectively. From the comparison analysis, the proposed method is achieving the best recall in every classes in human walking style-based emotion. The 
comparison analysis of precision is presented in figure 12. From the figure 12, the proposed method is achieved the 0.98 rate F_Measure in class 1 . Similarly, the MA-PSO and PSO-RNN methods are achieved the F_Measure 0.77 and 0.76 respectively. From the comparison analysis, the proposed method is achieving the best F_Measure in every classes in human walking style-based emotion. From the comparison analysis, the proposed method is achieving the best F_Measure in every classes in human walking style-based emotion detection. ROC curve, regression and error rate of the proposed method is illustrated in figure 13-15. From the comparison analysis, the proposed method is achieved the best results in the human walking style-based emotion detection by using the images.

\section{Conclusion}

In this paper, ADBNN-BWO Algorithm has been developed to identify the human emotions by human walking style images. This proposed methodology has been working based on four stages such as pre-processing, feature extraction, feature selection and classification. For the pre-processing, contrast enhancement median filter is used and Hu Moments, GLCM, F-SIFT, and skeleton features are used for the feature extraction. To extract the features efficiently, the feature extraction algorithm can be often very essential calculation. After that, feature selection is performed. Then the classification process has been done by utilizing the proposed ADBNN-BWO Algorithm. Based on the proposed method, the human gait recognition has been achieved which utilized to identify the emotions from the walking style. The proposed method is validated by using the open source gait databases. The proposed method is implemented in MATLAB platform and their corresponding performances/outputs are evaluated. Moreover, the statistical measures of proposed method are also determined and compared with the existing method as ANN, MAPSO, RNN-PSO and ANFIS respectively. In future, the proposed method will be applied in various emotions with different datasets.

\section{References}

[1]. Rijun Liao, Shiqi Yu, Weizhi An and Yongzhen Huang, "A model-based gait recognition method with body pose and human prior knowledge", Pattern Recognition, (2020) 98:107069.

[2]. Wenzheng Chi, Jiaole Wang and Max Q-H. Meng, "A gait recognition method for human following in service robots", IEEE Transactions on Systems, Man, and Cybernetics: Systems, (2017) 48(9):1429-1440. 
[3]. Guanqun Liu, Shaohui Zhong and Ting Li, "Gait recognition method of temporal-spatial HOG features in critical separation of Fourier correction points", Future Generation Computer Systems, (2019) 94:11-15.

[4]. Noriko Takemura, Yasushi Makihara, Daigo Muramatsu, Tomio Echigo and Yasushi Yagi, "Multi-view large population gait dataset and its performance evaluation for cross-view gait recognition", IPSJ Transactions on Computer Vision and Applications, (2018) 10(1):1-14.

[5]. Jiande Sun, Yufei Wang, Jing Li, Wenbo Wan, De Cheng and Huaxiang Zhang, "View-invariant gait recognition based on kinect skeleton feature", Multimedia Tools and Applications, (2018) 77(19):24909-24935.

[6]. Yanlin Li, Dexiang Zhang, Jun Zhang, Lina Xun, Qing Yan, Jingjing Zhang, Qingwei Gao and Yi Xia, "A convolutional neural network for gait recognition based on plantar pressure images", In Chinese Conference on Biometric Recognition, Springer, Cham, 2017 (2017):466-473.

[7]. Xiuhui Wang and Wei Qi Yan, "Human gait recognition based on frame-by-frame gait energy images and convolutional long short-term memory", International journal of neural systems, (2020) 30(01):1950027.

[8]. Francesco Battistone and Alfredo Petrosino, "TGLSTM: A time based graph deep learning approach to gait recognition", Pattern Recognition Letters, (2019) 126:132-138.

[9]. Munif Alotaibi and Ausif Mahmood, "Improved gait recognition based on specialized deep convolutional neural network", Computer Vision and Image Understanding, (2017) 164:103-110.

[10]. Xiaopeng Zhu, Lijun Yun, Feiyan Cheng and Chunjie Zhang, "LFN: Based on the Convolutional Neural Network of Gait Recognition Method", In Journal of Physics: Conference Series, (2020) 1650(3):032075.

[11]. Nurul Illiani Yaacob and Nooritawati Md Tahir, "Feature selection for gait recognition", In 2012 IEEE Symposium on Humanities, Science and Engineering Research, (2012):379-383.

[12]. Gao Fa-rong, Wang Jia-jia, Xi Xu-gang, She Qing-shan and Luo Zhi-zeng, "Gait recognition for lower extremity electromyographic signals based on PSO-SVM method", Journal of Electronics \& Information Technology, (2015) 37(5):1154-1159.

[13]. Mehmood, Asif, Muhammad Attique Khan, Muhammad Sharif, Sajid Ali Muhammad Shaheen Khan, Tanzila Saba, Naveed Riaz and Imran Ashraf, "Prosperous Human Gait Recognition: An end-to-end system based on pretrained CNN features selection", Multimedia Tools and Applications, (2020):1-21. 
[14]. Omaima N. Ahmad AL-Allaf and Shahlla A. AbdAlKader, "Performance Analysis of Different Feature Extraction Algorithms Used with Particle Swarm Optimization for Gait Recognition System", International Journal of Recent Technology and Engineering (IJRTE), (2015) 4(2):23-30.

[15]. Ujjawal Dixit, Apoorva Mishra, Anupam Shukla and Ritu Tiwari, "Texture classification using convolutional neural network optimized with whale optimization algorithm", SN Applied Sciences, (2019) 1(6):1-11.

[16]. Sanjeevkumar Angadi and Suvarna Nandyal, "Human identification system based on spatial and temporal features in the video surveillance system", International Journal of Ambient Computing and Intelligence (IJACI), (2020) 11(3):1-21.

[17]. Arshad. H, Khan. M, Sharif. M, Yasmin. M, Javed. M, "Multi-level features fusion and selection for human gait recognition: an optimized framework of Bayesian model and binomial distribution", International Journal of Machine Learning and Cybernetics, (2019) 10(12):3601-3618.

[18]. Seokeon Choi, Jonghee Kim, Wonjun Kim and Changick Kim, "Skeleton-based gait recognition via robust frame-level matching", IEEE Transactions on Information Forensics and Security, (2019) 14(10):2577-2592.

[19]. Qin Zou, Yanling Wang, Qian Wang, Yi Zhao and Qingquan Li, "Deep learning-based gait recognition using smartphones in the wild", IEEE Transactions on Information Forensics and Security, (2020) 15:3197-3212.

[20]. Hanqing Chao, Kun Wang, Yiwei He, Junping Zhang and Jianfeng Feng, "GaitSet: Cross-view Gait Recognition through Utilizing Gait as a Deep Set", IEEE Transactions on Pattern Analysis and Machine Intelligence, (2021) :1-1. [21]. Gajanand and Gupta, "Algorithm for image processing using improved median filter and comparison of mean, median and improved median filter", International Journal of Soft Computing and Engineering, (2011) 1(5):304-311. [22]. P.Mohanaiah, P. Sathyanarayana and L. GuruKumar, "Image texture feature extraction using GLCM approach", International journal of scientific and research publications, (2013) 3(5):1-5.

[23]. Liang-Chi and Chiu, Tian-Sheuan Chang, Jiun-Yen Chen and Nelson Yen-Chung Chang, "Fast SIFT design for real-time visual feature extraction", IEEE Transactions on Image Processing, (2013) 22(8):3158-3167.

[24]. Diogo Carbonera Luvizon, Hedi Tabia and David Picard, "Learning features combination for human action recognition from skeleton sequences", Pattern Recognition Letters, (2017) 99:13-20.

[25]. Maged Nasser, Naomie Salim, Hentabli Hamza, Faisal Saeed and Idris Rabiu, "Improved Deep Learning Based Method for Molecular Similarity Searching Using Stack of Deep Belief Networks", Molecules, (2021) 26(1):128. 
[26]. Essam H Houssein, Bahaa El-din Helmy, Diego Oliva, Ahmed A. Elngar, and Hassan Shaban,"A novel Black Widow Optimization algorithm for multilevel thresholding image segmentation", Expert Systems with Applications, (2021) 167:114159.

[27]. Shilpa Suresh, Jagalingam Pushparaj, Asha Cs, Shyam Lal, and Chintala Sudhakar Reddy, "Dehazing of Satellite Images using Adaptive Black Widow Optimization-based framework", International Journal of Remote Sensing, (2021) 42(13):5072-5090.

[28].http://gamma.cs.unc.edu/GAIT/\#: :text=We\%20also\%20present\%20an\%20\%22EWalk,from\%20videos\%20of $\% 20$ walking\%20individuals 